Moving forward with new data and approaches: a fresh look at anthracyclines in non-Hodgkin's lymphoma

\section{Dino Amadori}

Istituto Scientifico Romagnolo per lo Studio e la Cura dei Tumori (I.R.S.T.), Meldola, Italy

\section{Abstract}

Anthracyclines have a central role in the management of non-Hodgkin's lymphoma (NHL). The cyclophosphamide, doxorubicin, vincristine, and prednisone (CHOP) treatment regimen has been the standard of care for more than 20 years. Further improvements have been made to the efficacy of this chemotherapy by reducing the dosing interval and adding rituximab to the regimen. A major limitation to the use of anthracyclines is the development of cardiotoxicity as a late adverse event. Strategies to reduce cardiac events include changes to the dosing schedule for doxorubicin, use of the chelating agent dexrazoxane and the use of liposome-encapsulated doxorubicin. This latter strategy has demonstrated good efficacy and reduced cardiotoxicity in patients with NHL, including those at risk of developing cardiac effects.

\section{Introduction}

Since their introduction in the 1960 s, the anthracyclines have gained a central role in the treatment of non-Hodgkin's lymphoma (NHL). In particular, the use of doxorubicin in the standard rituximab, cyclophosphamide, doxorubicin, vincristine, and prednisone (RCHOP) regimen means that a large proportion of patients with aggressive lymphomas receive anthracycline therapy. In this paper, a brief history of the development of anthracyclines, their use in NHL, and the management of anthracycline-related cardiotoxicity are presented.

\section{Development of anthracyclines}

The potential of anthracyclines was first recognized in 1960, when Federico Arcamone and co-workers filed a patent application for the "fermentative production and recovery of an antibiotic obtained from Streptomyces krestomyceticus". In 1963, a novel cytotoxic compound derived from $S$. peuceticus was isolated simultaneously by researchers in Italy and France. The Italian group gave the compound the name daunomycin, the French group named it rubidomycine. Elements from both names were then combined to give the compound the name daunorubicin. A hydroxylated derivative of daunorubicin was subsequently isolated by the Italian group and called adriamycin. It was later renamed doxorubicin to conform to the established naming convention. In preclinical studies, this new antitumor antibiotic was shown to have greater activity against solid murine tumors and a higher therapeutic index than daunorubicin.1,2 Early clinical evaluation of doxorubicin demonstrated its efficacy in both adults and children with acute and chronic leukemias. ${ }^{2}$

The first key demonstration of the efficacy of CHOP in lymphoma was published in 1984. Armitage and co-workers reported the results of a clinical trial in 75 patients treated with CHOP for diffuse histiocytic lymphoma; 51\% achieved complete remission and 23 of the complete responders were alive and in complete remission at a median of 38 months after stopping treatment. ${ }^{3}$ Ten years later, the Southwest Oncology Group and the Eastern Cooperative Oncology Group published the results of a prospective Phase III trial comparing chemotherapy regimens commonly used at that time to treat advanced NHL. ${ }^{4}$ CHOP was therefore compared with MACOP-B (methotrexate with leucovorin rescue, doxorubicin, cyclophosphamide, vincristine, prednisone, and bleomycin), ProMACE-CytaBOM (prednisone, doxorubicin, cyclophosphamide, etoposide, followed by cytarabine, bleomycin, vincristine, methotrexate with leucovorin rescue), and m-BACOD (methotrexate with leucovorin rescue, bleomycin, doxorubicin, cyclophosphamide, vincristine, and dexamethasone) in 1138 patients (899 eligible for efficacy analysis). There were no significant differences in the efficacy of these regimens, but CHOP was associated with improved safety and tolerability compared with the other regimens (Figure 1). The authors therefore concluded that $\mathrm{CHOP}$ remains the best available treatment for patients with advanced-stage intermediate-grade or high-grade NHL. ${ }^{4}$ The efficacy of CHOP in the standard 21-day treatment cycle could be further improved by the use of a 14-day treatment cycle with granulocyte-colony stimulating factor (G-CSF) support in both young and elderly patients. ${ }^{5,6}$ Subsequently, it was found that the addition of rituximab to the CHOP regimen further increased its efficacy, $7-10$ and that six cycles of R-CHOP plus two additional cycles of rituximab were as effective as eight cycles of R-CHOP.11

\section{History of anthracycline-related cardiotoxicity}

Early studies of doxorubicin did not identify cardiotoxicity as an adverse event. Although the
Correspondence: Professor Dino Amadori, Istituto Scientifico Romagnolo per lo Studio e la Cura dei Tumori (I.R.S.T.) S.r.l., via Piero Maroncelli, 40 47014 Meldola (FC), Italy.

Tel. +39.0543.739912 - Fax: +39.0543 .739249 .

E-mail: segronco@ausl.fo.it

Key words: anthracycline, non-Hodgkin's lymphoma, doxorubicin, liposomal doxorubicin.

Conflict of interest: the author has received honoraria from Cephalon.

This work is licensed under a Creative Commons Attribution NonCommercial 3.0 License (CC BYNC 3.0).

(C) Copyright D. Amadori, 2011

Licensee PAGEPress, Italy

Hematology Reports 2011; 3(s3):e1

doi:10.4081/hr.2011.s3.e1

Phase I and II studies did demonstrate electrocardiographic changes in more than half of 97 patients who were prospectively studied, these abnormalities were subclinical, no symptoms were mentioned, and additional research did not suggest any cumulative cardiac toxicity of doxorubicin. 12 However, 10 years after these first studies, cardiotoxicity started to be identified as a late side-effect of cumulative doses of anthracyclines. An early analysis of this problem demonstrated a correlation between the cumulative dose of doxorubicin and the probability of developing congestive heart failure (CHF) (Figure 2).13 Subsequent studies confirmed that the incidence of CHF increased from $3 \%$ at a cumulative doxorubicin dose of $400 \mathrm{mg} / \mathrm{m}^{2}$ to $18 \%$ at a cumulative dose of $700 \mathrm{mg} / \mathrm{m}^{2} .{ }^{14}$ Moreover, CHF was often irreversible. Risk factors for cardiotoxicity included: previous exposure to anthracyclines, patient age, and a history of cardiovascular disease. ${ }^{4}$ Cardiotoxicity is also seen with newer anthracyclines, such as epirubicin. ${ }^{15}$ It is the main factor limiting anthracycline use, and can result in discontinuation or non-use of treatment for patients who might otherwise benefit from this therapy.

\section{Managing anthracycline cardiotoxicity}

Treatment strategies designed to reduce anthracycline cardiotoxicity have included modification of the dosing regimen, treatment with cardioprotective agents, and the use of liposome-encapsulated forms of doxorubicin.

\section{Dose and schedule modification}

As higher cumulative doses of doxorubicin are associated with a greater risk of $\mathrm{CHF}$, then maintaining doses below a maximum cumulative dose may reduce patients' risks. ${ }^{16}$ Nevertheless, such maximum doses cannot be used 
alone as safe limits for drug treatment, as cardiac dysfunction has been seen in patients receiving lower than maximal doses, and some patients are more susceptible to cardiotoxicity than others.13,16 Changes to the administration of the anthracycline can also alter its toxicity. Thus, long drug infusions instead of a single injection have been shown to be less cardiotoxic. 17,18 However, this approach is more laborious than conventional treatment, particularly when doxorubicin is used in combination with other agents.

\section{Use of dexrazoxane}

Several studies have investigated the use of dexrazoxane, an iron-chelating agent that scavenges free radicals, as a means of limiting anthracycline-related cardiotoxicity. Initially, there were concerns that this agent might adversely affect the antitumor activity of doxorubicin. ${ }^{19}$ However, a subsequent systematic review of nine randomized trials found that dexrazoxane significantly reduced patients' risk of developing CHF, without affecting the efficacy of doxorubicin, in terms of response rate or survival. The authors therefore concluded that dexrazoxane treatment should be considered in patients at high risk of cardiotoxicity. ${ }^{20}$

\section{Use of liposome-encapsulated doxorubicin}

Two liposome-encapsulated forms of doxorubicin are currently available: pegylated liposomal doxorubicin and non-pegylated liposomal doxorubicin (NPLD). Liposomes are microscopic structures consisting of one or more concentric lipid bilayers, which encapsulate the active substance. These lipid structures cannot escape the vascular space in areas that have tight capillary junctions, such as the heart muscle, but can reach other tissues. Thus, the pharmacokinetics and tissue distribution of the active molecule are modified. In particular, NPLD is preferentially delivered to the liver, spleen, bone marrow, and lymph nodes, compared with relatively little entry to cardiac muscle and the gastrointestinal mucosa. ${ }^{21}$ This treatment may therefore reduce the cardiac toxicity of doxorubicin while maintaining its antitumor efficacy.

In patients with newly diagnosed aggressive NHL, substitution of NPLD into the CHOP regimen, to give COMP, produced a complete response rate of $67.4 \% .22$ Importantly, the use of COMP in patients at risk of cardiotoxicity has also produced promising results. A retrospective analysis of rituximab-COMP (RCOMP) treatment for patients who either had concurrent cardiac diseases or had been pretreated with anthracyclines demonstrated a complete response rate of $76 \% .23$ Moreover, only one patient included in this analysis developed acute grade III cardiotoxicity. A prospective international study of R-COMP in elderly patients with aggressive lymphoma gave a complete remission rate of $57 \%$, and patients showed no signs of cardiotoxicity. Left ventricular ejection fraction did not change during treatment, and the R-COMP regimen was not associated with any increase in acute cardiac toxicity. ${ }^{24} \mathrm{~A}$ meta-analysis of two studies comparing free and liposomal doxorubicin (in patients with breast cancer, not NHL) showed a significantly lower rate of both clinical heart failure, and clinical and subclinical heart failure combined, in patients treated with liposome-encapsulated doxorubicin. 15

\begin{tabular}{|cccc}
\hline & $\begin{array}{c}\text { Patients } \\
\text { at risk }\end{array}$ & Deaths & $\begin{array}{c}\text { 3-year } \\
\text { estimate (\%) }\end{array}$ \\
\hline CHOP & 225 & 88 & 54 \\
m-BACOD & 223 & 93 & 52 \\
- ProMACE-CytaBOM & 233 & 97 & 50 \\
MACOP-B & 218 & 93 & 50 \\
& & $\mathrm{P}=0.90$ & \\
\hline
\end{tabular}
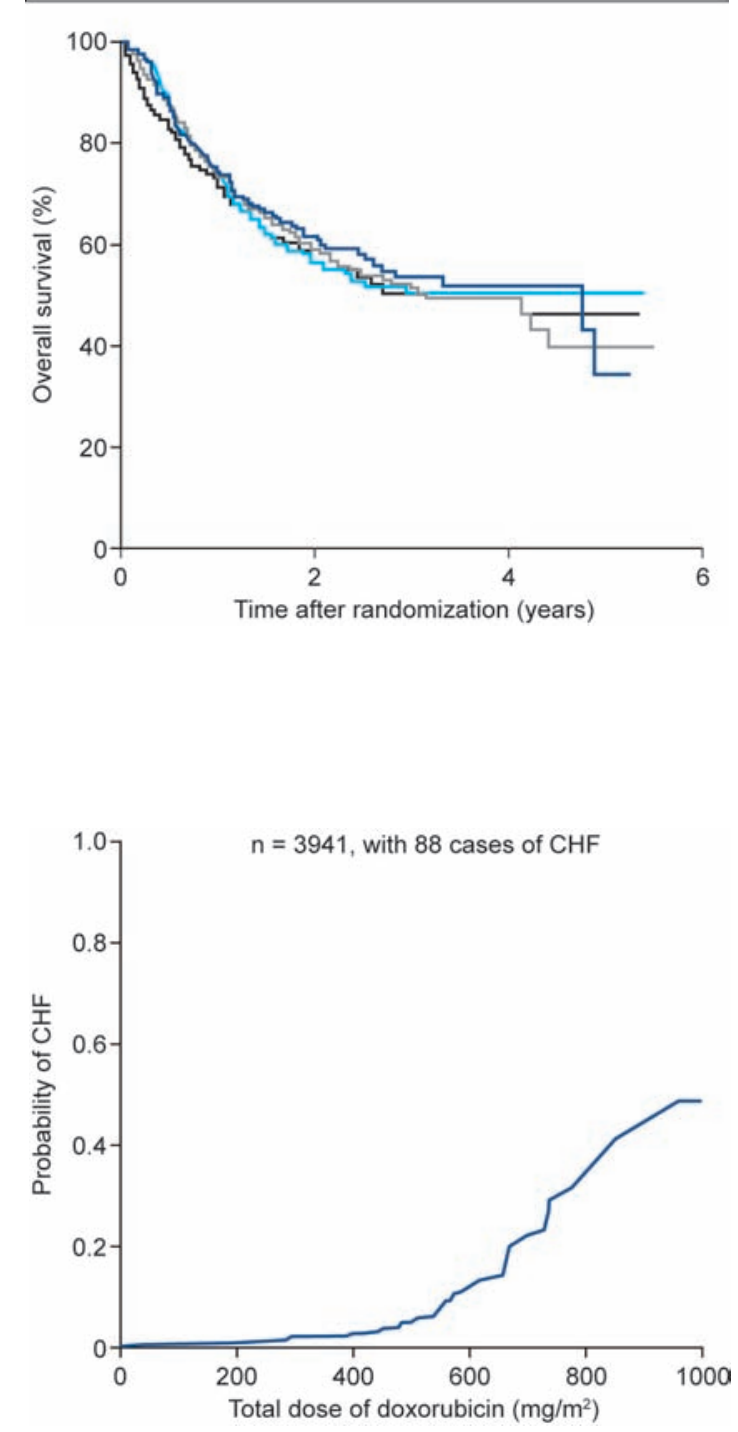

[Hematology Reports 2011; 3(s3):e1]

\section{Conclusions}

Anthracyclines continue to play a central role in the treatment of NHL. Moreover, the introduction of liposome-encapsulated doxorubicin may allow their use to be extended to patients at risk of cardiotoxicity who would benefit from this treatment. A key challenge now in NHL is to maximize treatment outcomes, through new biological approaches such as gene profiling, and through optimal use of therapies across the diverse spectrum of lymphomas. This challenge to clinicians was the theme of an international workshop, Moving forward with new data and approaches: a fresh
Figure 1. Overall survival of patients with non-Hodgkin's lymphoma receiving three different treatment regimens (3year estimates of survival). ${ }^{4}$ CHOP - cyclophosphamide, doxorubicin, vincristine, prednisone; m-BACOD - methotrexate with leucovorin rescue, bleomycin, doxorubicin, cyclophosphamide, vincristine, and dexamethasone; ProMACECytaBOM - prednisone, doxorubicin, cyclophosphamide, etoposide, followed by cytarabine, bleomycin, vincristine, methotrexate with leucovorin rescue; MACOP-B - methotrexate with leucovorin rescue, doxorubicin, cyclophosphamide, vin cristine, prednisone, and bleomycin. Reprinted with permission from Fisher RI et al. Comparison of a standard regimen (CHOP) with three intensive chemotherapy regimens for advanced non-Hodgkin's lymphoma. N Engl J Med 1993;328 1002-6. ${ }^{\circ}$ Massachusetts Medical Society.

Figure 2. Risk of developing congestive heart failure (CHF) in relation to the cumulative dose of doxorubicin. 13 Reprinted with permission from Von Hoff DD et al. Risk factors for doxorubicin-induced congestive heart failure. Ann Intern Med 1979; 91:710-17. 
look at anthracyclines in breast cancer and $N H L$, which took place in Meldola, Italy, in September 2010. This meeting reviewed recent data on the use of anthracyclines in the treatment of breast cancer and NHL, with the aim of providing guidance on how the management of these diseases can be improved. The presentations on NHL at this meeting are summarized in this supplement.

\section{References}

1. Di Marco A, Gaetani M, Scarpinato B. Adriamycin (NSC-123,127): a new antibiotic with antitumor activity. Cancer Chemother Rep 1969;53:33-7.

2. Bonadonna G, Monfardini S, De Lena M, Fossati-Bellani F. Clinical evaluation of adriamycin, a new antitumour antibiotic. Br Med J 1969;3:503-6.

3. Armitage J0, Fyfe MA, Lewis J. Long-term remission durability and functional status of patients treated for diffuse histiocytic lymphoma with the CHOP regimen. J Clin Oncol 1984;2:898-902.

4. Fisher RI, Gaynor ER, Dahlberg S, et al. Comparison of a standard regimen (CHOP) with three intensive chemotherapy regimens for advanced nonHodgkin's lymphoma. N Engl J Med 1993; 328:1002-6.

5. Pfreundschuh M, Trumper L, Kloess M, et al. Two-weekly or 3-weekly CHOP chemotherapy with or without etoposide for the treatment of elderly patients with aggressive lymphomas: results of the NHL-B2 trial of the DSHNHL. Blood 2004; 104:634-41.

6. Pfreundschuh M, Trumper L, Kloess M, et al. Two-weekly or 3-weekly CHOP chemotherapy with or without etoposide for the treatment of young patients with goodprognosis (normal LDH) aggressive lymphomas: results of the NHL-B1 trial of the DSHNHL. Blood 2004;104:626-33.

7. Coiffier B, Lepage E, Briere J, et al. CHOP chemotherapy plus rituximab compared with CHOP alone in elderly patients with diffuse large-B-cell lymphoma. N Engl $\mathrm{J}$ Med 2002;346:235-42.

8. Pfreundschuh M, Trumper L, Osterborg A, et al. CHOP-like chemotherapy plus rituximab versus CHOP-like chemotherapy alone in young patients with good-prognosis diffuse large-B-cell lymphoma: a randomised controlled trial by the MabThera International Trial (MInT) Group. Lancet Oncol 2006;7:379-91.

9. Sehn LH, Donaldson J, Chhanabhai M, et al. Introduction of combined CHOP plus rituximab therapy dramatically improved outcome of diffuse large B-cell lymphoma in British Columbia. J Clin Oncol 2005; 23:5027-33.

10. Habermann TM, Weller EA, Morrison VA, et al. Rituximab-CHOP versus CHOP alone or with maintenance rituximab in older patients with diffuse large B-cell lymphoma. J Clin Oncol 2006;24:3121-7.

11. Pfreundschuh M, Schubert J, Ziepert M, et al. Six versus eight cycles of bi-weekly CHOP-14 with or without rituximab in elderly patients with aggressive CD20+ Bcell lymphomas: a randomised controlled trial (RICOVER-60). Lancet Oncol 2008;9: 105-16.

12. Bonadonna G, Monfardini S, De Lena M, Fossati-Bellani F, Beretta G. Phase I and preliminary phase II evaluation of adriamycin (NSC 123127). Cancer Res 1970; 30:2572-82.

13. Von Hoff DD, Layard MW, Basa P, et al. Risk factors for doxorubicin-induced congestive heart failure. Ann Intern Med 1979; 91:710-17.

14. Hortobagyi GN. Anthracyclines in the treatment of cancer. An overview. Drugs 1997;54 Suppl 4:1-7.

15. van Dalen EC, Michiels EM, Caron HN, Kremer LC. Different anthracycline derivates for reducing cardiotoxicity in cancer patients. Cochrane Database Syst Rev 2006:CD005006.

16. Swain SM, Whaley FS, Ewer MS. Conges- tive heart failure in patients treated with doxorubicin: a retrospective analysis of three trials. Cancer 2003;97:2869-79.

17. Legha SS, Benjamin RS, Mackay B, et al. Reduction of doxorubicin cardiotoxicity by prolonged continuous intravenous infusion. Ann Intern Med 1982;96:133-9.

18. van Dalen EC, van der Pal HJ, Caron HN, Kremer LC. Different dosage schedules for reducing cardiotoxicity in cancer patients receiving anthracycline chemotherapy. Cochrane Database Syst Rev 2009: CD005008.

19. Speyer JL, Green MD, Zeleniuch-Jacquotte A, et al. ICRF-187 permits longer treatment with doxorubicin in women with breast cancer. J Clin Oncol 1992;10:117-27.

20. van Dalen EC, Caron HN, Dickinson HO, Kremer LC. Cardioprotective interventions for cancer patients receiving anthracyclines. Cochrane Database Syst Rev 2008: CD003917.

21. Swenson CE, Perkins WR, Roberst $P$, Janoff AS. Liposome technology and the development of MyocetTM (liposome doxorubicin citrate). The Breast 2001;10 Suppl 2:1-7.

22. Tulpule A, Espina BM, Berman N, et al. Phase I/II trial of nonpegylated liposomal doxorubicin, cyclophosphamide, vincristine, and prednisone in the treatment of newly diagnosed aggressive nonHodgkin's lymphoma. Clin Lymphoma Myeloma 2006;7:59-64.

23. Rigacci L, Mappa S, Nassi L, et al. Liposome-encapsulated doxorubicin in combination with cyclophosphamide, vincristine, prednisone and rituximab in patients with lymphoma and concurrent cardiac diseases or pre-treated with anthracyclines. Hematol Oncol 2007;25:198-203.

24. Luminari S, Montanini A, Caballero D, et al. Nonpegylated liposomal doxorubicin (Myocet ${ }^{\mathrm{TM}}$ ) combination (R-COMP) chemotherapy in elderly patients with diffuse large B-cell lymphoma (DLBCL): results from the phase II EUR018 trial. Ann Oncol 2010;21:1492-9. 